\title{
The reverse Wessely phenomenon: immune corneal rings following systemic immunisation
}

\author{
NORMAN T. FELBERG AND THEODORE W. SERY \\ From the Wills Eye Research Institute, Philadelphia, Pennsylvania, USA
}

SUMMARY Rabbits were hyperimmunised systemically over a 4-week period with bovine serum albumin (BSA) in complete Freund's adjuvants and were subsequently challenged systemically with a massive dose of BSA designed to produce serum sickness. Without any ocular manipulation a spontaneous corneal immune reaction was observed which we termed a 'reverse Wessely phenomenon'. It was due to a reversed sequence of antibody becoming sequestered in the cornea and later responding to antigen entering via the limbal circulation. In the stroma, lymphocytes were prominent and only few polymorphonuclear cells were found, but this may have been due to late sectioning of the corneas tested. The corneal ring formed at the limbus and migrated centripetally ahead of peripheral clouding, which is typical of the Wessely phenomenon. Possible relationships of this reaction with some autoimmune disorders are discussed.

Since the time when Wessely (1911) first demonstrated an immune reaction to foreign serum protein introduced into the rabbit cornea several other related immune phenomena have been described. Morawiecki (1956) demonstrated that the cornea could behave much like an agar substrate for migration of immune reactants in producing precipitin arcs as in gel diffusion. Parks et al. (1962) revealed a delayed hypersensitivity response in the cornea if it was overloaded with antigen in attempts to produce the Wessely phenomenon. The cornea was used for many years as the critical in-vivo test for delayed hypersensitivity to a given antigen (Salvin and Gregg, 1961). Sery et al. $(1962,1971)$ and Sery and Nagy (1971) showed that after recovering from an initial antigen sensitisation the cornea possessed an immune memory, independent of humoral immune factors, expressed as a local immune phenomenon for as long as 1 year after challenge. The same effect, described as 'super rings' by Breebaart and James-Witte (1959), was thought to be merely an amplified Wessely phenomenon, but the antigen diffusion kinetics and other characteristics were shown to be distinct (Sery and Nagy, 1962).

The Wessely phenomenon may be considered to be a type of Arthus phenomenon lacking only the

Address for reprints: Dr N. T. Felberg, Wills Eye Research Institute, 1601 Spring Garden Street, Philadelphia, Pennsylvania 19130 , USA characteristics of focal necrosis, but this is essentially a problem in terminology. The Arthus reaction depends on the presence, or close proximity, of blood capillaries which, on becoming clogged with immune precipitates and neutrophils, deprive the local tissue of nutrients. These vessels are lacking in the normal cornea. Thus attempts by Waksman and Bullington (1956) to demonstrate a passive Arthus reaction in the cornea failed for anatomical reasons. However, immune ring precipitates followed by influx of polymorphonuclear leucocytes which initiate the Arthus reaction in vascularised tissues do occur in the cornea (Waksman and Bullington, 1956; Salvin and Gregg, 1961; Sery et al., 1962; Rahi and Tripathi, 1976).

The present study demonstrates another expression of the Wessely phenomenon which can be best explained as a 'reverse Wessely phenomenon', because antibody becomes sequestered in the cornea from the circulation during systemic immunisation and is manifested at a later time by systemic injection of a very large dose of that antigen. Thus, the immune precipitin reaction of the Wessely is reversed in the order which the immune reactants enter the cornea. Because this response is spontaneous in the normal cornea, which receives no direct manipulation, it may be comparable to corneal rings occasionally seen in patients with autoimmune disease with soluble immune complexes entering the cornea. 


\section{Methods}

Thirty-four $2 \cdot 5-\mathrm{kg}$ New Zealand white or chinchilla rabbits of both sexes were employed in these studies. They were sensitised intradermally on the back to bovine serum albumin (BSA, Sigma Chemical Co., St. Louis, Mo.) $1 \mathrm{mg} / 0 \cdot 1 \mathrm{ml}$ mixed with an equal volume of complete Freund's adjuvant on days 0, 7 , and 14.

On day 21 the rabbits were challenged intraperitoneally with $250 \mathrm{mg}$ of BSA (as a sterile $35 \%$ solution). Rabbits that did not develop corneal opacities within 2 weeks were rechallenged intravenously on days 41 and 112 with $250 \mathrm{mg}$ of BSA.

Before sensitisation rabbit corneas were examined by biomicroscopy and transillumination and shown to be free of opacities and patent vessels. After challenge rabbits were examined daily during the first week, then every other day for the second week, and twice weekly thereafter.

Rabbit serum antibody was estimated by passive haemagglutination of sheep red blood cells conjugated to BSA with glutaraldehyde (Avrameas et al., 1969). Titres were recorded as $\log _{2}$.

Rabbits were killed by an overdose of pentobarbitone and the eyes fixed in buffered formalin. Paraffin sections were stained with haematoxylin and eosine.

Various unfixed tissues of the eye were tested for the presence of antibody after extraction. Corneas were removed with a trephine to within $2 \mathrm{~mm}$ of the limbus. Each cornea (60 to $70 \mathrm{mg}$ wet weight) was ground in a mortar with $1 \mathrm{~g}$ of silica and 10 volumes of saline. After storage at $4^{\circ} \mathrm{C}$ overnight, the mixture was centrifuged and the antibody in the supernatant estimated by passive haemagglutination. In a similar manner lenses were extracted with 4 volumes of saline for antibody determinations.

\section{Results}

\section{RING FORMATION}

During the sensitisation period no eye changes were observed. After the first antigen challenge $(250 \mathrm{mg}$ BSA intraperitoneally) 12 corneas in 9 rabbits developed corneal rings (Table 1). The earliest immune rings were seen 2 days after antigen challenge (Fig. 1). While the latest response occurred 13 days after challenge, the median response was seen by day 4. Corneal rings were first visible as a diffuse peripheral haze, randomly located along approximately one-third of the limbal margin. By the next morning a fine white arc was discernible. A white translucent area could be seen between the arc and the limbus. Gradually over a period of 1 to 2 days the arcs coalesced into rings as they
Table 1 Incidence of reverse Wessely phenomenon with method and number of challenge

\begin{tabular}{|c|c|c|c|c|}
\hline & $\begin{array}{l}\text { Challeng } \\
1 \text { st }\end{array}$ & $\begin{array}{l}\text { mber } \\
2 n d\end{array}$ & $3 r d$ & Total \\
\hline Intraperitoneal & $12 / 42 *$ & $2 / 2$ & $0 / 0$ & $14 / 44$ \\
\hline Intravenous & $1 / 4$ & $4 / 12$ & $5 / 8$ & $10 / 24$ \\
\hline Total & $13 / 46$ & $6 / 14$ & $5 / 8$ & $24 \nmid / 68$ \\
\hline
\end{tabular}

* Number of corneas developing ring arcs per total corneas in experimental group. $\uparrow 24$ corneas in 16 rabbits

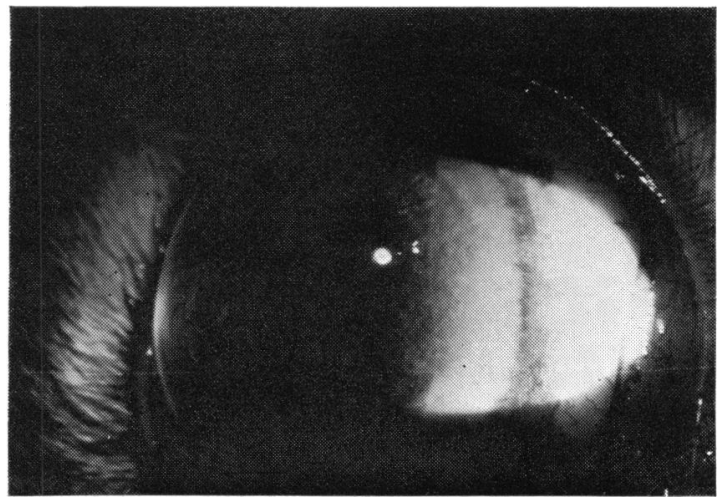

Fig. 1 Corneal ring and translucent area seen 2 days after antigen challenge

migrated towards the centre of the cornea. The translucent area continued to occupy the area between the corneal rings and the limbus. After 1 week faint corneal rings continued, but the haze had cleared. Within 2 weeks the corneal ring had also cleared (Fig. 2). Not all eyes developed complete rings. Many formed arcs of from $90^{\circ}$ up to $270^{\circ}$.

One rabbit received a $250-\mathrm{mg}$ intraperitoneal antigen challenge of fluorescein-conjugated BSA on day 21 , and the next day had a fluorescent line at the limbus of the left eye. By the second day green fluorescent arcs were detected with long wavelength ultraviolet light in both eyes, but the arcs were not visible by transillumination with white light.

\section{ANTIBODY DETECTION}

After 3 weekly antigen sensitisations specific antibody could be detected in cornea, aqueous humour, and serum but not in lens or vitreous humour (Table 2). While the amount of antibody in most of the corneas was 6 twofold dilutions lower (i.e., $1: 64)$, corneal and serum titres were almost identical in 1 rabbit (No. 3193, Table 2). The variation in antibody titre for 2 rabbits developing rings during the course of the experiment is shown in Fig. 3. 
A
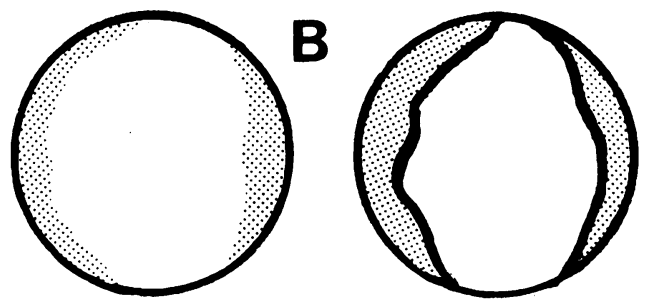

C
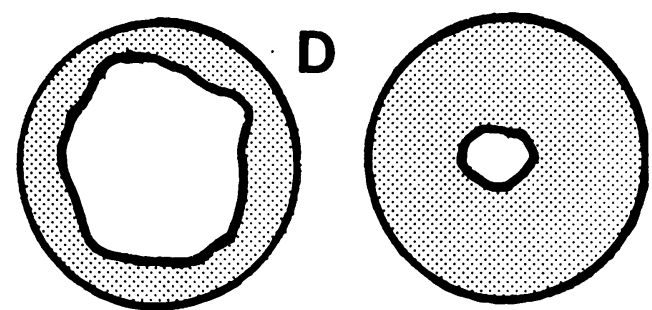

Fig. 2 Diagrammatic development of reverse Wessely corneal rings. (A) Initial peripheral haze. (B) Fine white corneal arcs preceding translucent haze to centre of cornea. (C) Arcs coalesce to form a continuous corneal ring with translucent area to limbus. (D) Translucent area fades, leaving faint white ring which can still be discerned after 1 week

Table 2 BSA antibodies in ocular tissues and serum

\begin{tabular}{|c|c|c|c|c|c|c|c|}
\hline \multirow{3}{*}{$\begin{array}{l}\text { Rabbit no } \\
\text { Eye }\end{array}$} & \multicolumn{7}{|c|}{ Passive haemagglutination titre $\left(\log _{2}\right)$} \\
\hline & & 3185 & & 3193 & 3212 & 3214 & 3216 \\
\hline & $O D$ & OS & $O D$ & os & $O D O S$ & $O D O S$ & $O D O S$ \\
\hline Cornea* & $8 \cdot 3$ & $8 \cdot 3$ & $7 \cdot 8$ & 6.9 & $5 \cdot 3$ & $5 \cdot 3$ & $6 \cdot 3<4 \cdot 3$ \\
\hline Aqueous & - & -- & - & - & $3 \cdot 0$ & 5.0 & $4.0 \quad 4.0$ \\
\hline Lens† & - & - & - & - & $<1 \cdot 0<1 \cdot 0$ & $<1.0<1.0$ & $<1.0<1.0$ \\
\hline Vitreous & - & - & 一 & - & $2 \cdot 0 \quad 1 \cdot 0$ & $<1 \cdot 0$ & $2 \cdot 0$ \\
\hline Serum $\downarrow$ & $14 \cdot 0$ & & 8.0 & & $11 \cdot 0$ & 13.0 & $12 \cdot 0$ \\
\hline Killed & D43 & & D43 & & D22 & D22 & D22 \\
\hline
\end{tabular}

*Corneas diluted 10-fold during homogenisation, except 3216 OS which was diluted 20 -fold. †Lenses diluted 4-fold during homogenisation. $\ddagger$ Rabbits sensitised on day 0,7 , and 14 , and serum obtained on the day killed (day 21)

\section{HISTOLOGY}

An extensive cellular infiltrate was found in the limbal region correlating with the clinical picture of rabbits having rings for several days. Infiltration up to $3 \mathrm{~mm}$ was occasionally seen, but no discrete histological process was seen at the demarcation of the corneal ring. The cellular infiltrate was composed of mononuclear leucocytes, which were predominantly lymphocytes. Occasional immature plasma cells and histiocytes were seen in the corneal stroma. Neutrophils were rarely seen in the corneal stroma, but small numbers could be found in the limbal region (Fig. 4).

\section{Discussion}

The Wessely phenomenon, or immune corneal rings, is the result of systemically formed antibody diffusing into a cornea containing residual antigen from a previous injection. We have referred to the study described in this report as the 'reverse' Wessely phenomenon, because we believe the antigen or a soluble immune complex of the antigen diffuses into a physiologically antibody-sensitised cornea.

Both models have several common properties. The corneal manifestations were almost identical, with initial peripheral corneal haze leading to sharply demarcated arcs or rings which migrate towards the centre of the cornea. Histologically, cellular infiltrates were an important facet of both phenomena. However, while the predominant infiltrative cell in the Wessely phenomenon is the neutrophil, there was a predominance of lymphocytes in the reverse Wessely phenomenon. Finding only rare neutrophils in the cornea during the active phase of ring formation and migration in the reverse Wessely phenomenon is probably the result of killing the animal after the neutrophil phase of ring formation had passed. A major difference between the 2 models lies in the production of immune corneal rings in the reverse Wessely phenomenon, without intraocular injection.

There are at least two possible mechanisms for the development of immune rings following the serumsickness-induced reverse Wessely phenomenon.

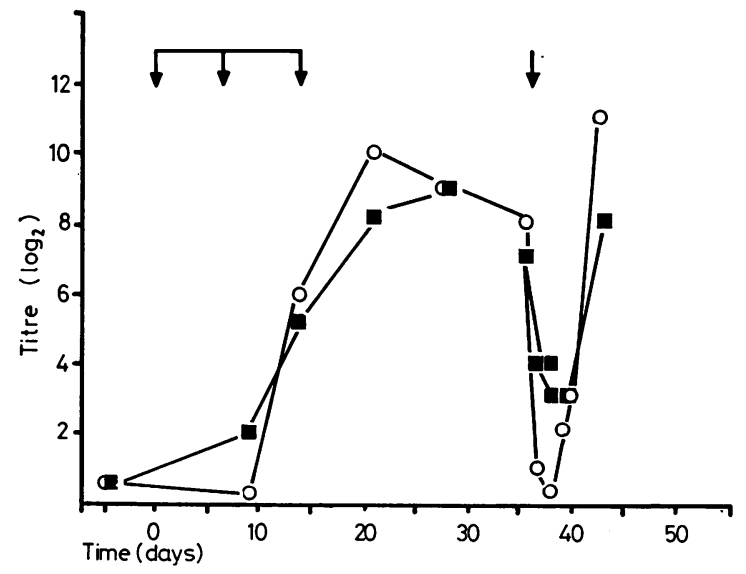

Fig. 3 Antibody titres in 2 rabbits developing immune corneal rings. Antigen sensitisation on days 0,7 , and 14 (first 3 arrows). Intravenous antigen challenge on day 36 (last arrow) with $250 \mathrm{mg}$ of $B S A$ 

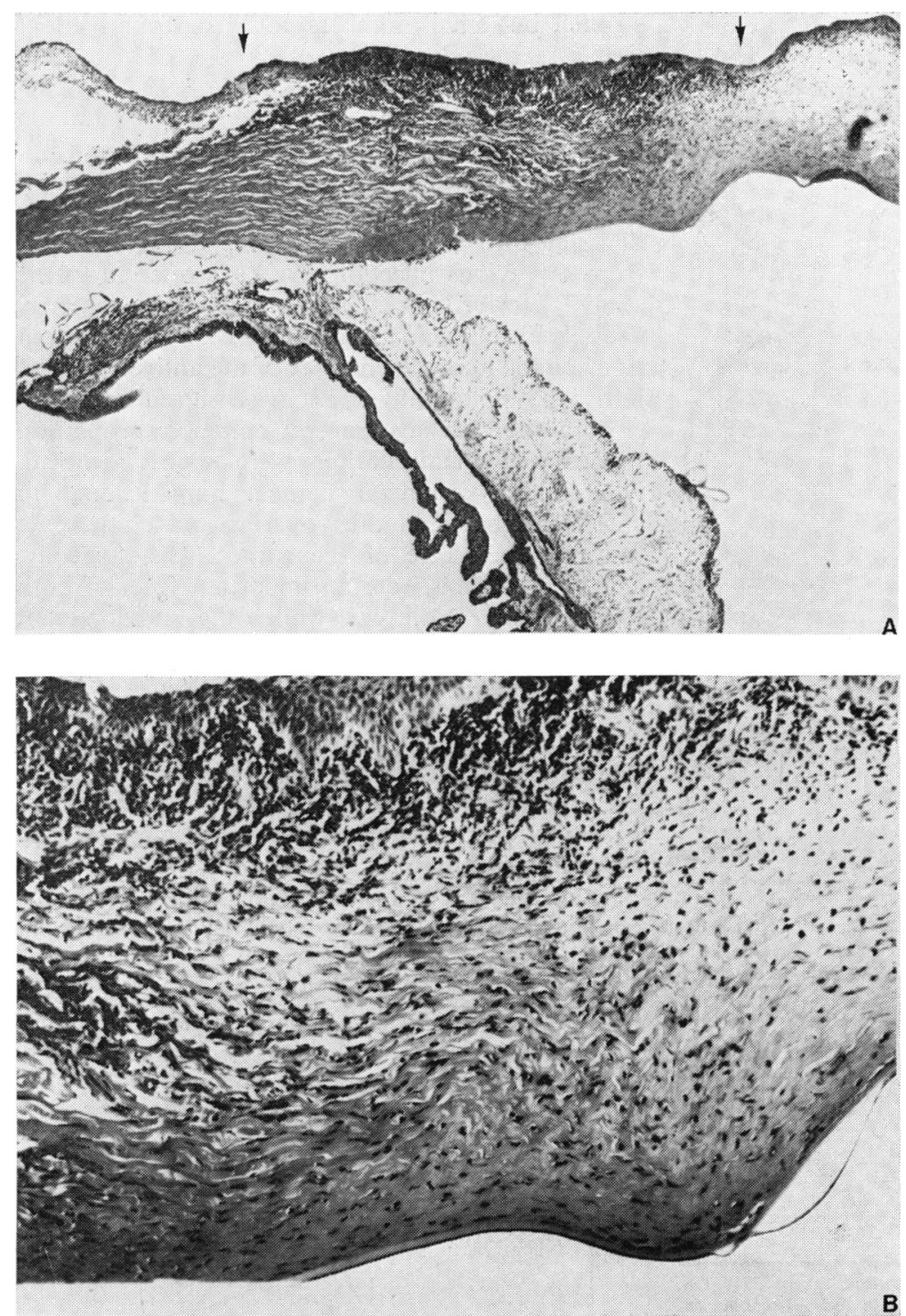

Fig. 4 Cellular infiltrate in the cornea of the rabbit shown in Fig. 1. (A) Mononuclear leucocyte infiltrate in the cornea (between arrows) extending approximately $2.5 \mathrm{~mm}$ from the limbus. $H$. and E., original magnification $\times 10$. (B) The lymphocyte is the predominant cell in the infiltrate. Occasional neutrophils were also seen in the cornea of this 4-dayold response. $H$. and $E$., original magnification $\times \mathbf{4 0}$
Immunoglobulin has been reported to be normally present in the cornea in a uniform concentration throughout the cornea (Allansmith et al., 1971). Specific antibody was repeatedly detected in the cornea of sensitised rabbits at considerable titres (Table 2). Firstly, soluble immune complexes or unbound antigen may enter the cornea through limbal circulation or the anterior chamber and be rendered insoluble by the sequestered antibody. The immune precipitates are followed by cellular influx and the model is complete. A severe immunogenic uveitis usually accompanies serum sickness
(Wong et al., 1971; Friedman, 1976). Antibodies can enter the cornea from the anterior chamber and form immune precipitates in the cornea (Rahi and Tripathi, 1976). Alternatively a second mechanism can be proposed independent of the presence of specific antibody in the cornea. Rahi and Garner (1976) suggest that immune complexes persist at the limbus because of the absence of corneal circulation. The influx and activation of complement at the limbus might change the permeability of the limbal vessels, allowing the rapid influx of leucocytes, giving rise to the corneal rings. At present neither mechan- 
ism is favoured over the other, and each may play a part in immune ring development.

The variability in the production of the reverse Wessely immune rings may be due to the variability in the development of serum sickness. For example, 2 rabbits not reported in this study were initially sensitised and challenged with antigen but died from serum sickness within several hours. Other rabbits in the study showed little or no uveitis, a finding suggesting reduced development of serum sickness. Serial antibody titres were not determined in all rabbits as described in Fig. 3, nor were complement levels measured, so that the degree of serum sickness in each animal was not known.

Several human autoimmune diseases have rare corneal manifestations such as marginal furrowing ulceration and peripheral opacification (Rahi and Garner, 1976; Ginsberg, 1976). It is possible that these corneal changes may occur by a mechanism similar to the reverse Wessely phenomenon. It is suggested that circulating immune complexes arrive at the limbus and remain because of a lack of corneal vascularisation. Activation of complement by the immune complex may trigger cellular infiltration, resulting in the appearance of immune rings.

In the serum-sickness-induced reverse Wessely phenomenon the cornea clears within 2 weeks, leaving no clinical evidence of opacification. In human immune complex disease, such as rheumatoid arthritis, polyarteritis nodosa, systemic lupus erythematosus, and relapsing polychondritis, occasional peripheral corneal opacification and ischaemic necrosis can be found (Rahi and Garner, 1976; Ginsberg, 1976). Continuous deposition of immune complexes at the limbus, along with $C^{\prime}$-mediated local tissue destruction and neutrophil infiltration, may be responsible for the marginal ulceration or ischaemic necrosis seen on occasion in patients with these autoimmune diseases.

This investigation was supported in part by Public Health Service Research Grants EY 00195, Training Grant EY 00084, and Academic Career Development Award from the National Eye Institute KO7-EY00032 (to NTF) and Biomedical Research Support Grant RR 05510 from the National Institutes of Health.

\section{References}

Allansmith, M., Newman, L., and Whitney, C. (1971). The distribution of immunoglobulin in the rabbit eye. Archives of Ophthalmology, 86, 60-64.

Avrameas, S., Taundon, B., and Chuilon, S. (1969). Glutaraldehyde, cyanuric chloride and tetraazotized O-dianisidine as coupling reagents in the passive hemagglutination test. Immunochemistry, 6, 67-76.

Breebaart, A. C., and James-Witte, J. (1959). Studies on experimental corneal allergy. American Journal of Ophthalmology, 48, 37-47.

Friedman, A. H. (1976). Ocular hypersensitivity. In Clinical Ophthalmology, vol. 5, chapter 28. Edited by T. D. Duane. Harper and Row: Hagerstown.

Ginsberg, S. P. (1976). Corneal problems in systemic disease. In Clinical Ophthalmology, vol. 5, chapter 43. Edited by T. D. Duane. Harper and Row: Hagerstown.

Morawiecki, J. (1956). Präzipitationserscheinungen in der lebenden Hornhaut bei Antigen-Antikorperraktionen. Ophthalmologica, 132, 236-243.

Parks, J. J., Leibowitz, H. M. I., and Maumenee, A. E. (1962). A transient stage of suspected delayed sensitivity during the early induction phase of immediate corneal sensitivity. Journal of Experimental Medicine, 115, 867880.

Rahi, A. H. S., and Garner, A. (1976). Immunopathology of the Eye, p. 131. Blackwell: Oxford.

Rahi, A. H. S., and Tripathi, R. C. (1976). Anatomy of passive arthus reaction in the cornea. Modern Problems in Ophthalmology, 16, 155-160.

Salvin, S. B., and Gregg, M. B. (1961). The specificity of allergic reaction. 4. The cornea. Proceedings of the Society for Experimental Biology and Medicine, 107, 478-483.

Sery, T. W., Pinkes, A. H., and Nagy, R. M. (1962). Immune corneal rings. 1. Evaluation of reactions to equine albumin. Investigative Ophthalmology, 1, 672-685.

Sery, T. W., and Nagy, R. M. (1962). Immune corneal rings. 3. Mechanism of local immune corneal ring formation. Investigative Ophthalmology, 1, 762-772.

Sery, T. W., Nagy, R. M., and Barber, G. W. (1971). Corneal hypersensitivity. 1. Escape kinetics of antigen from sensitized corneas. Journal of Immunology, 106, 217-222.

Sery, T. W., and Nagy, R. M. (1971). Corneal hypersensitivity. 2. Specific binding of antigen in sensitized corneas. Journal of Immunology, 106, 226-232.

Waksman, B. H., and Bullington, S. J. (1956). A quantitative study of the passive Arthus reactions in the rabbit eye. Journal of Immunology, 76, 441-453.

Wessely, K. (1911). Ueber anaphylaktische Erscheinungen an der Hornhaut (experimentelle Erzeugung einer parenchymatosen Keratitis durch artfremdes Serum). Münchener medizinische Wochenschrift, 58, No. 32.

Wong, V. G., Anderson, R. R., and McMaster, P. R. B. (1971). Endogenous immune uveitis. The role of serum sickness. Archives of Ophthalmology, 85, 93-102. 